\title{
Who's Holding Up the Queue? Delay in Treatment of Rheumatoid Arthritis
}

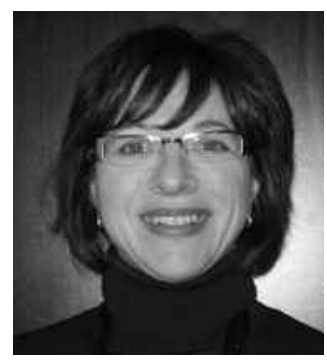

Much emphasis has been placed on early diagnosis and treatment of rheumatoid arthritis (RA) in order to optimize outcomes for our patients. Numerous studies have identified the clinical and radiological benefits of early initiation of disease-modifying antirheumatic drugs (DMARD) $)^{1,2,3,4,5,6}$, and new American College of Rheumatology/European League Against Rheumatism (ACR/EULAR) classification criteria for RA appear aimed at identifying RA earlier ${ }^{7}$. Unfortunately, the practicalities of achieving the goal of early treatment depend on optimal performance of the medical system. There are many hurdles to overcome before a patient with joint symptoms presents to a rheumatologist. First, the patient has to recognize that they have a medical problem that warrants mention to their primary healthcare provider (PCP) and obtain an appointment. We can call this patient delay. Next, the PCP has to recognize that the patient may have an inflammatory arthritis that may benefit from referral to a specialist and complete that referral (PCP delay). Then there may be significant wait time to see the rheumatologist, depending on whether there are early arthritis clinics available, or triage systems in place, to facilitate rapid assessment (rheumatologist delay).

Shiff, et $a l^{8}$ examined the time to referral of pediatric patients in a prospective multicenter Canadian juvenile idiopathic arthritis (JIA) cohort. They examined geographic barriers (distance to specialist), presenting clinical features, family history, ethnicity, and parental education. Fever, South Asian ethnicity, and parental education level were most predictive in their model. An accompanying editorial by Foster and Rapley ${ }^{9}$ categorized the barriers to referral as physical factors (clinical features), recognition of JIA (prolonged investigation by other specialists such as an orthopedic surgeon), healthcare provision (geographic distance to specialist), and sociocultural factors (as an influence on health-seeking behavior).

Van der Linden, et $a l^{10}$ examined the ramifications of delayed assessment by specialists for patients with RA in their Leiden Early Arthritis Clinic cohort and categorized the delay as being patient-related or GP-related. It was felt that once a patient was assessed by a rheumatologist, there was rapid initiation of DMARD. Factors that appeared to influence delay in assessment by the specialist were mostly clinical features of the disease such as insidious onset and normal acute-phase reactants, symmetry, and small-joint involvement, although that might be due to the priority given to patients with reactive arthritis, crystal-induced arthritis, or sarcoidosis. The identification of older age and female sex as significant factors may work through influence on health-seeking behavior. An accompanying editorial by Bykerk and Emery ${ }^{11}$ highlights the twin issues of patient awareness of a problem worth mentioning and primary care awareness of a problem worth referring. It also underlines the importance of adverse clinical outcomes such as radiologic progression as a result of delays in assessment.

Data from the ESPOIR cohort of patients with early arthritis in France revealed that the mean time to see a primary care provider and rheumatologist was 26.3 and 75.5 days, respectively ${ }^{12}$. Factors associated with more rapid rheumatology assessment were acute or subacute onset, persistent joint involvement, fever, or direct access to a rheumatologist appointment (no longer supported through the national health insurance system).

A similar cohort of newly diagnosed patients with RA in 19 centers in the UK and Eire was analyzed for delays in access to DMARD treatment ${ }^{13}$. The authors were unable to comment on patient delay, but significant delay was seen from symptom-onset to PCP referral to a rheumatologist (median 4 months) and symptom-onset to first DMARD (median 8 months). Median wait time to see a rheumatologist was 1 month, and median time to first DMARD from rheumatologist appointment was also 1 month.

There appears to be consistency among most publications that rapid initiation of DMARD therapy for both RA and JIA is occurring when patients are seen by the rheumatology specialist. This reflects the successful dissemination of the results of years of research showing the longterm effects of delayed treatment in RA. There is also a consistent message of delay in referring patients to a rheumatologist by the primary care specialist and less delay due to

See Time to treatment for new RA patients in a major metropolitan city, page 1282 
rheumatologist wait times in these published studies. This consistency remained despite the fact that the studies were done in 4 different countries.

In this issue of The Journal, Jamal, et al ${ }^{14}$ report on a retrospective cohort study of newly diagnosed patients with RA from 15 rheumatologists in the Greater Toronto Area. Through telephone interviews and retrospective chart reviews, date of symptom-onset and date of DMARD initiation were determined. The results show roughly $22 \%$ of patients initiating DMARD within 3 months and $48 \%$ of patients initiating DMARD within 6 months. The authors note that this result is similar to that in other recent studies, with older studies from the late 1990s finding longer median time to DMARD initiation. The median time to DMARD initiation once a rheumatologic assessment has been done was 0 months. The timely initiation of DMARD by the rheumatologist is consistent with previous studies in this area.

In a diagram of the time delays expressed as medians for the cohort, Jamal, et al show there is a 3-month delay in symptom-onset to rheumatology referral, most of that being accounted for by PCP delay and relatively little patient delay. Time from rheumatology referral to DMARD initiation is 2 months, most of that due to the rheumatology wait time, and in some cases delay in RA diagnosis.

The authors then conducted a multivariate logistic regression analysis of predictors of early initiation of DMARD (3 months). The variables used can be grouped as patient characteristics, clinical characteristics, and rheumatologist characteristics. The only significant predictor was swollen joint count at presentation to the rheumatologist.

Some authors have found patient delay to be significant $^{15}$, others less so ${ }^{10}$. Several studies have identified patient characteristics such as female sex and older age as delaying presentation to the rheumatologist based on both patient and PCP delay ${ }^{10,16}$, although that was not a factor in this analysis or in other cohorts ${ }^{15}$. Jamal, et al actually report a trend toward significance of increased age and higher education in predicting earlier treatment.

Their analysis explored characteristics of rheumatologist practice demographics, which did not appear to influence the delay to treatment. This study was conducted over the Greater Toronto Area, which would have a defined specialist density within the referral region and may not be comparable to other regions in North America. One previous study found specialist density to be a factor contributing to referral times ${ }^{12}$. A missed opportunity in this and previous studies is an analysis of primary care provider characteristics, which might contribute to our understanding of referral practices and further guide interventions to improve the referral process.

The landscape has changed regarding the management of inflammatory arthritis, with great strides being made to ensure early detection and institution of therapy. The new
ACR/EULAR guidelines aim to identify disease at an earlier stage and there is abundant evidence that earlier treatment results in better outcomes. There appears to be successful dissemination of this new management paradigm, as evidenced by the many studies that report early institution of DMARD once the patient is seen by a rheumatologist. Although the studies cited above did not report significant wait times to see a specialist, this is likely still a problem, and strategies to effectively triage patients with inflammatory arthritis need to be evaluated and implemented. Bykerk and Emery refer to an ACR/EULAR task force being formed to explore this aspect of the referral delay ${ }^{11}$. As suggested by Jamal, et al, continued efforts at educating physicians regarding the assessment of arthritis symptoms and signs are required. Finally, educating the public regarding the significance of their arthritis symptoms and necessity for early diagnosis and treatment may enhance "health-seeking behaviors" and shorten the delays to treatment.

\author{
JOANNE E. HOMIK, MD, \\ Department of Medicine, \\ University of Alberta, \\ 562 Heritage Medical Research Building, \\ University of Alberta, \\ Edmonton, Alberta T6G 2S2, Canada
}

Address correspondence to Dr.Homik; E-mail: joanne.homik@ualberta.ca

\section{REFERENCES}

1. Mottonen T, Hannonen P, Korpela M, Nissila M, Kautiainen H, Ilonen J, et al. Delay to institution of therapy and induction of remission using single-drug or combination disease-modifying antirheumatic drug therapy in early rheumatoid arthritis. Arthritis Rheum 2002;46:894-8.

2. Lard L, Visser H, Speyer I, vander Horst-Bruinsma I, Zwinderman A, Breedveld F, et al. Early versus delayed treatment in patients with recent-onset rheumatoid arthritis: comparison of two cohorts who received different treatment strategies. Am J Med 2001;111:446-51.

3. Goekoop-Ruitermam Y, De Vries-Bouwstra J, Allart C, Van Zeben D, Kerstens P, Hazes J, et al. Clinical and radiographic outcomes of four different treatment strategies in patients with early rheumatoid arthritis (the BeSt study): A randomized, controlled trial. Arthritis Rheum 2005;52:3381-90.

4. Quinn M, Conaghan P, O’Connor P, Karim Z, Greenstein A, Brown $\mathrm{A}$, et al. Very early treatment with infliximab in addition to methotrexate in early, poor-prognosis rheumatoid arthritis reduces magnetic resonance imaging evidence of synovitis and damage, with sustained benefit after infliximab withdrawal: Results from a twelve-month randomized, double-blind, placebo-controlled trial. Arthritis Rheum 2005;52:27-35.

5. Bejarano V, Quinn M, Conaghan P, Reece R, Keenan A-M, Walker $\mathrm{D}$, et al. Effect of the early use of the anti-tumor necrosis factor adalimumab on the prevention of job loss in patients with early rheumatoid arthritis. Arthritis Care Res 2008;59:1467-74.

6. Nell V, Machold K, Eberl G, Stamm T, Uffmann M, Smolen J. Benefit of very early referral and very early therapy with disease-modifying anti-rheumatic drugs in patients with early rheumatoid arthritis. Rheumatology 2004;43:906-14.

7. Aletaha D, Neogi T, Silman A, Funovits J, Felson D, Bingham C, et 
al. 2010 Rheumatoid arthritis classification criteria: An American College of Rheumatology/European League Against Rheumatism collaborative initiative. Arthritis Rheum 2010;62:2569-81.

8. Shiff N, Tucker L, Guzman J, Oen K, Yeung R, Duffy C. Factors associated with a longer time to access pediatric rheumatologists in Canadian children with juvenile idiopathic arthritis. J Rheumatol 2010;37:2415-21.

9. Foster H, Rapley T. Access to pediatric rheumatology care - a major challenge to improving outcome in juvenile idiopathic arthritis. J Rheumatol 2010;37:2199-201.

10. van der Linden M, le Cessie S, Raza K, van der Woude D, Knevel $\mathrm{R}$, Huizinga T, et al. Long-term impact of delay in assessment of patients with early arthritis. Arthritis Rheum 2010;62:3537-46.

11. Bykerk V, Emery P. Delay in receiving rheumatology care leads to long-term harm. Arthritis Rheum 2010;62:3519-21.

12. Fautrel B, Benhamou M, Foltz V, Rincheval N, Rat A-C, Combe B, et al. Early referral to the rheumatologist for early arthritis patients: evidence for suboptimal care. Results from the ESPOIR cohort. Rheumatology 2010;49:147-55.
13. Kiely P, Williams R, Walsh D, Young A. Contemporary patterns of care and disease activity outcome in early rheumatoid arthritis: the ERAN cohort. Rheumatology 2009;48:57-60.

14. Jamal S, Alibhai S, Badley E, Bombardier C. Time to treatment for new rheumatoid arthritis patients in a major metropolitan city. J Rheumatol 2011;38:1282-8.

15. Kumar K, Daley E, Carruthers D, Situnayake D, Gordon C, Grindulis K, et al. Delay in presentation to primary care physicians is the main reason why patients with rheumatoid arthritis are seen late by rheumatologists. Rheumatology 2007;46:1438-40.

16. Palm $\varnothing$, Purinszky E. Women with early rheumatoid arthritis are referred later than men. Ann Rheum Dis 2005;64:1227-8.

J Rheumatol 2011;38:1225-7; doi:10.3899/jrheum.110649 